\title{
Long-term variations of vector and tensor anisotropies of cosmic rays
}

\author{
P.Yu. Gololobov*, G.F. Krymsky, P.A. Krivoshapkin \\ Yu.G. Shafer insitute of cosmophysical research and aeronomy of SB RAS, Yakutsk, Russia \\ E-mail: gpetereikfia.sbras.ru, krymskyeikfia.sbras.ru, \\ p.a.krivoshapkin@mail.ru
}

On the basis of data of Nagoya multi-directional muon telescope the long-term variations of vector and tensor anisotropies of cosmic rays that was registered in the period 1971-2015 are investigated. Decomposition of observed diurnal anisotropy into vector and tensor components is held using the method of receiving vectors. It is shown, that: 1. the amplitude of vector anisotropy reveals 11-year variations and the phase - 22-year, which is influenced by the 11-year solar activity cycle; 2 . the antisymmetric diurnal component of tensor anisotropy experiences sustainable annual oscillations along the hour line 9-21 of local time, and the amplitude of which manifests 11 -year variations and at the solar maxima reaches the value $0.1 \% ; 3$. the semidiurnal component of tensor anisotropy undergoes annual and semiannual oscillations with amplitude $0.1 \%$. The amplitude and phase of this component reveals 11 - and 22-year variations, respectively. It is shown that the obtained average characteristics of tensor anisotropy can be described within the frameworks of the models of cosmic ray screening by sectoral interplanetary magnetic field and the shear flow of solar wind. The reasons for the appearance of 11- and 22-year variations in the tensor anisotropy is discussed. The origin of annual and semiannual variations of tensor anisotropy is due to the shift of interplanetary magnetic field to the south of the solar equatior and non-coincidence of the axes of rotation of the Earth and the Sun.

35th International Cosmic Ray Conference - ICRC2017

10-20 July, 2017

Bexco, Busan, Korea

* Speaker. 


\section{Introduction}

In the works $[1,2]$ it was established that the anisotropy of cosmic rays (CR) in the solar system is appeared due to an anisotropic diffusion in the spiral magnetic field. This diffusion compensates a removal of CR by solar wind and directed toward the solar system. This simple picture subsequently was supplemented by an idea of magnetic drift of particles [3] which explains a 22-year modulation of CR anistropy. Thus the average characteristics of CR anisotropy can be described by the convective diffusion theory. The theory is responsible for first angular distribution of CR asymmetric diurnal variation. However during last years investigations of second angular distribution of CR $[4,5]$ have revealed the existence of antisymmetric diurnal variations that can not be explained within the frameworks of the mentioned theory. The current work is devoted to the investigation of this kind of CR anisotropy.

\section{Investigation method}

Observed CR anisotropy can presented as a decomposition of the first two spherical harmonics. The first spherical harmonic consists of the components $\left(a_{1}^{0}, a_{1}^{1}, b_{1}^{1}\right)$ and can be represented as a vector in a certain way oriented in the space. The second spherical harmonic with components $\left(a_{2}^{0}\right.$, $a_{2}^{1}, b_{2}^{1}, a_{2}^{2}, b_{2}^{2}$ ) is more complex and can be represented in the space as a some tensor ellipsoid. Therefore the first and second spherical harmonics are named as vector and tensor anisotropies of $\mathrm{CR}$, respectively. The most common oscillations of the CR intensity during a day are a symmetric diurnal $R_{1}^{1}$ and semidiurnal $R_{2}^{2}$, which are symmetric relative to the Earth equator. Moreover, it exists antisymmetric diurnal variation $R_{2}^{1}$ that is a contribution of the second spherical harmonic into the first one. A pair of stations which are located in the opposite hemispheres of the Earth would registed the $R_{2}^{1}$ as a diurnal oscillations of CR intensity with the same amplitude but opposite phases.

In the current work in order to define the vector and tensor anisotropies of CR we have used the data of multidirectional muon telescope of st. Nagoya $\left(35.09^{\circ} \mathrm{N}, 136.58^{\circ} \mathrm{S}\right)$ that operates since 1970 till nowadays (the data are available through the link ${ }^{1}$ ). The detector registers CR from 17 independent directions and cover a huge area of celestial sphere and allow us to study the angular distribution of CR in details.

The flow of CR that are registered by CR detectors is influenced by the geomagnetic field, particularly, the magnetic field bends the trajectories of charged particles. The problem can be effectively solved with applying so-called receiving vectors. In the current work we used the receiving vectors that are presented in [6].

Observed angular distribution of CR $I(\theta, \phi)$ can be represented in the space as spherical harmonics:

$$
I(\theta, \phi)=\sum_{n=0}^{\infty} \sum_{m=0}^{n}\left(a_{n}^{m} \cos (m \phi)+b_{n}^{m} \sin (m \phi)\right) P_{n}^{m}(\sin \theta),
$$

where $\theta$ and $\phi$ are latitude and longitude, $P_{n}^{m}(\sin \phi)$ is associated Legendre polynomials. If to represent the distribution $I(\theta, \phi)$ as a multidimensional vector $\vec{A}=\left(a_{n}^{m}, b_{n}^{m}\right)$ then for each device

\footnotetext{
${ }^{1}$ http://www.stelab.nagoya-u.ac.jp/ste-www1/div3/muon/muon1.html
} 
we can define a multidimensional receiving vector $\vec{Z}=\left(x_{n}^{m}, y_{n}^{m}\right)$ so, that the registered intensity $J=\vec{A} \cdot \vec{Z}$.

Calculating of a receiving vector $\vec{Z}$ requires accounting of directional diagrams $N(\theta, \phi)$, coupling coefficients $W(E)$, CR particles asymptotic arrival angles $\Psi, \Phi$ and a spectrum of observed anisotropy $f_{n}(E)$. In the current work we have used the receiving vectors that are presented in work [6] with the suggestion of spectrum for CR diurnal variation as

$$
f_{n}(E)=\left\{\begin{array}{ll}
(E / 10 G V)^{\gamma}, & \text { if } E \leq E_{0} \\
0 & \text { if } E>E_{0}
\end{array},\right.
$$

where $\gamma=0$ and $E_{0}=100 \mathrm{GV}$ for the vector anisotropy and $\gamma=0.5$ and $E_{0}=100 \mathrm{GV}$ for the tensor anisotropy.

When the number of devices (channels) are enough the vector $\vec{A}$ can be found by solving the system of linear equations, which number is equals to the number of devices. Below the multidimentional vector $\vec{A}$ definition process is presented.

Let us decompose the observed intensity $J_{i}$ on 24- and 12-hour harmonics. Then for the $i$-th station (channel) we would have: $J_{i}=\left(a_{1, i}, b_{1, i}, a_{2, i}, b_{2, i}\right)$, where $a_{1, i}, b_{1, i}$ and $a_{2, i}, b_{2, i}$ are diurnal and semidiurnal components of Fourier decomposition, respectively.

At the same time for the same $i$-th station we have corresponding receiving vector: $\vec{Z}_{i}=$ $\left(x_{1, i}^{1}, y_{1, i}^{1}, x_{2, i}^{1}, y_{2, i}^{1}, x_{2, i}^{2}, y_{2, i}^{2}\right)$.

Let us compose matrices of receiving vectors $\mathbf{M}_{\mathbf{1}}$ and $\mathbf{M}_{\mathbf{2}}$ in the following way:

$$
\mathbf{M}_{\mathbf{1}}=\left(\begin{array}{cccc}
x_{1,1}^{1} & y_{1,1}^{1} & x_{2,1}^{1} & y_{2,1}^{1} \\
-y_{1,1}^{1} & x_{1,1}^{1} & -y_{2,1}^{1} & x_{2,1}^{1} \\
\vdots & \vdots & \vdots & \vdots \\
x_{1, j}^{1} & y_{1, j}^{1} & x_{2, j}^{1} & y_{2, j}^{1} \\
-y_{1, j}^{1} & x_{1, j}^{1} & -y_{2, j}^{1} & x_{2, j}^{1}
\end{array}\right), \mathbf{M}_{2}=\left(\begin{array}{cc}
x_{2,1}^{2} & y_{2,1}^{2} \\
-y_{2,1}^{2} & x_{2,1}^{2} \\
\vdots & \vdots \\
x_{2, j}^{2} & y_{2, j}^{2} \\
-y_{2, j}^{2} & x_{2, j}^{2}
\end{array}\right),
$$

and also the vectors of observed diurnal and semidiurnal variations:

$$
\mathbf{A}_{\mathbf{1}, \mathbf{o b s}}=\left(\begin{array}{c}
a_{1,1} \\
b_{1,1} \\
\vdots \\
a_{1, j} \\
b_{1, j}
\end{array}\right), \mathbf{A}_{\mathbf{2 , o b s}}=\left(\begin{array}{c}
a_{2,1} \\
b_{2,1} \\
\vdots \\
a_{2, j} \\
b_{2, j}
\end{array}\right)
$$

And the vectors of the expected symmetric and antysimmetric diurnal $\mathbf{A}_{\mathbf{1} \text {,exp }}$ and semidiurnal $\mathbf{A}_{\mathbf{2} \text {,exp }}$ components of the vector and tensor anisotropies would have the form:

$$
\mathbf{A}_{\mathbf{1}, \mathbf{e x p}}=\left(\begin{array}{c}
a_{1}^{1} \\
b_{1}^{1} \\
a_{2}^{1} \\
b_{2}^{1}
\end{array}\right), \mathbf{A}_{\mathbf{2}, \mathbf{e x p}}=\left(\begin{array}{c}
a_{2}^{2} \\
b_{2}^{2}
\end{array}\right)
$$

Finally, we will have the next matrix equations: 


$$
\begin{aligned}
& \mathbf{A}_{1, \mathrm{obs}}=\mathbf{M}_{1} \mathbf{A}_{1, \exp }, \\
& \mathbf{A}_{\mathbf{2}, \mathrm{obs}}=\mathbf{M}_{\mathbf{2}} \mathbf{A}_{\mathbf{2}, \mathbf{e x p}},
\end{aligned}
$$

and transforming them into the form:

$$
\begin{aligned}
& \left(\mathbf{M}_{1}^{\mathrm{T}} \mathbf{M}_{1}\right)^{-\mathbf{1}} \mathbf{M}_{1}^{\mathrm{T}} \mathbf{A}_{1, \mathrm{obs}}=\mathbf{A}_{\mathbf{1}, \exp }, \\
& \left(\mathbf{M}_{\mathbf{2}}^{\mathrm{T}} \mathbf{M}_{\mathbf{2}}\right)^{-\mathbf{1}} \mathbf{M}_{\mathbf{2}}^{\mathrm{T}} \mathbf{A}_{\mathbf{2}, \mathrm{obs}}=\mathbf{A}_{\mathbf{2}, \mathbf{e x p}},
\end{aligned}
$$

we can find the components of vector $a_{1}^{1}, b_{1}^{1}$ and tensor $a_{2}^{1}, b_{2}^{1}, a_{2}^{2}, b_{2}^{2}$ anisotropies of CR. In order to calculate the errors of the components of vectors $\mathbf{A}_{\mathbf{1}, \mathbf{e x p}}$ and $\mathbf{A}_{\mathbf{2}, \mathbf{e x p}}$ let us insert the next divergence matrices

$$
\triangle_{1}=\mathbf{A}_{1, \mathrm{obs}}-\mathbf{M}_{1} \mathbf{A}_{1, \mathrm{exp}}, \triangle_{2}=\mathbf{A}_{2, \mathrm{obs}}-\mathbf{M}_{\mathbf{2}} \mathbf{A}_{2, \mathrm{exp}}
$$

The mean square deviations of the measured components will be equal to

$$
\sigma_{1}=\frac{\triangle_{1}^{T} \triangle_{1}}{N_{1}-n_{1}}, \sigma_{2}=\frac{\triangle_{2}^{T} \triangle_{2}}{N_{2}-n_{2}}
$$

where $N_{1}, N_{2}$ stands for the number of channels (detectors) in the matrices $\mathbf{M}_{1}, \mathbf{M}_{\mathbf{2}}$, respectively. $n_{1}, n_{2}$ denotes the number of unknown quantities in the vectors $\mathbf{A}_{\mathbf{1} \text {,exp }}$ and $\mathbf{A}_{\mathbf{2} \text {,exp }}$.

\section{Analysis of the obtained data and results}

Using the method of receiving vectors by the data of multidirectional muon telescope st. Nagoya we have obtained average monthly values of parameters of vector $\left(a_{1}^{1}, b_{1}^{1}\right)$ and tensor $\left(a_{2}^{1}, b_{2}^{1}, a_{2}^{2}, b_{2}^{2}\right)$ anisotropies of CR for the time period since 1971 to 2015 . The results of the calculations are presented in Figure 1. Preliminary analysis of the obtained data has revealed presence of stable seasonal oscillations in the components of tensor anisotropy of CR. The components of vector anisotropy do not experience such changes. In order to study a long-term dependence of the obtained anisotropy parameters we have determined average yearly amplitudes and phases of the components of vector and tensor anisotropies of CR which are presented in figure 2 . In the same picture the solar sunspot number and the average solar field strength of the Northern and Southern Hemispheres, that is taken by measurements from the Wilcox Solar Observatory ${ }^{2}$ are shown. As seen from the figure, the amplitudes of symmetric diurnal $\left|\vec{R}_{1}^{1}\right|$ semidiurnal $\left|R_{2}^{2}\right|$ variations of CR experiences 11-year variations with solar activity, during the minima of solar activity the value $\left|R_{1}^{1}\right|$ decreases to $0.14 \%$ and $\left|R_{2}^{2}\right|$ - to $0.03 \%$ and during the other periods they are equals in average to $0.4 \%$ and $0.1 \%$, respectively. In contrast to them, the mean annual value of $\left|R_{2}^{1}\right|$ does not reveals the same changes and has in average amplitude only $0.02 \%$. Moreover, it is seen that the phase of symmetric diurnal $T_{1, \max }^{1}$ and semidiurnal $T_{2, \max }^{2}$ variations experiences 22-yearly oscillations in dependence with polarity of the general magnetic field of the Sun (GMFS). In the solar activity minima periods in case of positive polarity of GMFS $T_{1, \max }^{1}$ shifts to the earlier time $\approx 10 \mathrm{~h}$ and in case of negative polarity shifts to the lesser earlier time $\approx 16 \mathrm{~h}$ and during the other periods it holds $\approx 18 \mathrm{~h}$ maximum time. At that, $T_{1, \max }^{1}$ and $T_{2, \max }^{2}$ correlates between each other very well. The same results concerning a diurnal variations were obtained in [7] by the data of st. Nagoya

\footnotetext{
${ }^{2}$ http://wso.stanford.edu/Polar.html
} 


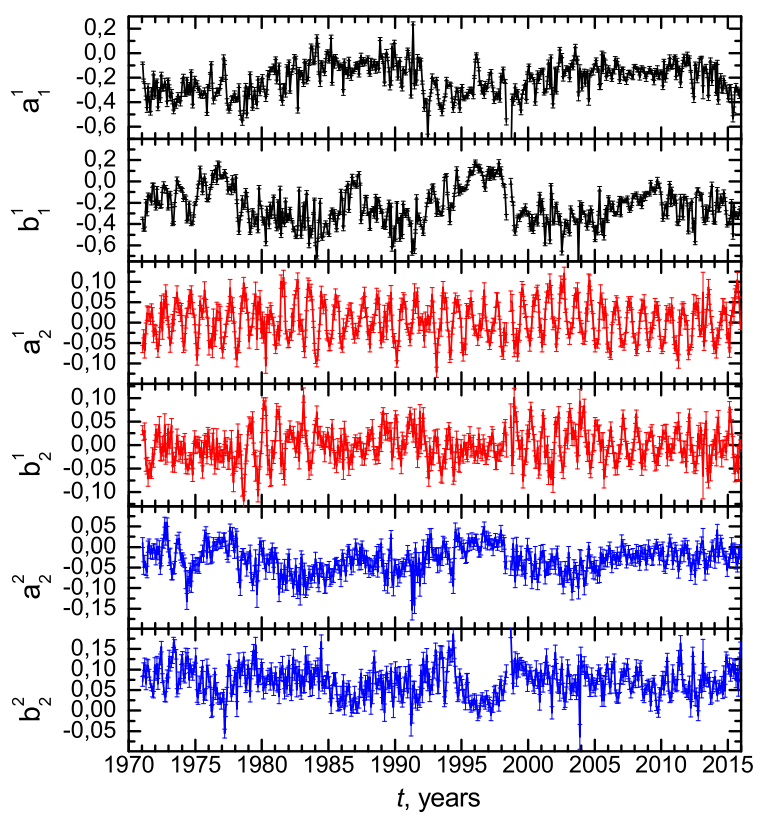

Figure 1: Observed mean monthly values of the component of diurnal $\left(a_{1}^{1}, b_{1}^{1}\right)$, antisymmetric $\left(a_{2}^{1}, b_{2}^{1}\right)$ and semidiurnal $\left(a_{2}^{2}, b_{2}^{2}\right)$ variations of $\mathrm{CR}$ which are obtained by the data of 17 directions of the multidirectional muon telescope st. Nagoya for the time period 1971-2017

for the time period 1970-2013. Moreover, in figure 2 also the long-term variations of the spread of the vector of antysimmetric diurnal variation during a year $\left|\triangle R_{2}^{1}\right|$ are shown. Which is also shows dependence with the solar activity cycle. Such dependence of the obtained parameters of vector and tensor anisotropies means that they are of solar origin. In total, the observed oscillations of the amplitude and phase of symmetric diurnal variation are common and can be explained within the frameworks convective-diffusion and drift motion of CR. CR drift direction change in the periods of opposite polarities of GMFS is the reason of the changes of symmetric diurnal variation. Insignificant short term shift of $T_{1, \max }^{1}$ to the earlier time can be explained by the influence of electrostatic field of the heliosphere, which shifts $T_{1, \max }^{1}$ to the earlier time during periods of solar activity minima regardless to the polarity of GMFS. The other reason can be concentration of the solar wind to low heliolatitudes during the periods of solar activity minima, which increases the convective removal of CR and, consequently, leads to the shift of $T_{1, \text { max }}^{1}$ to earlier time.

In average, during the whole considered period, the phase of semidiurnal variation $T_{2, \max }^{2}$ is equals to $\approx 3 \mathrm{~h} \mathrm{LT}$, that can be explained by a CR screening mechanism [9], which creates a deficit of particles moving along the interplanetary magnetic field. As seen from the figure 2, against the background of $3 \mathrm{~h}$ value, $T_{2, \max }^{2}$ reveals 22-year variations that correlates to $T_{2, \max }^{2}$. Such behavior of $T_{2, \max }^{2}$ can not be explained within the screening mechanism and the further investigations are required.

As was mentioned before, $\vec{R}_{2}^{1}$ and $\vec{R}_{2}^{2}$ experience stable annual and semiannual oscillations. In figure 3 on a hour dials the observed on st. Nagoya vectors of antisymmetric diurnal $\vec{R}_{2}^{1}$ and semidiurnal $\vec{R}_{2}^{2}$ variations, which are averaged during the period 1971-2015, are presented. It is seen that $\vec{R}_{2}^{1}$ oscillates during a year strictly around the line $9-21 \mathrm{~h}$, moreover during the autumn 


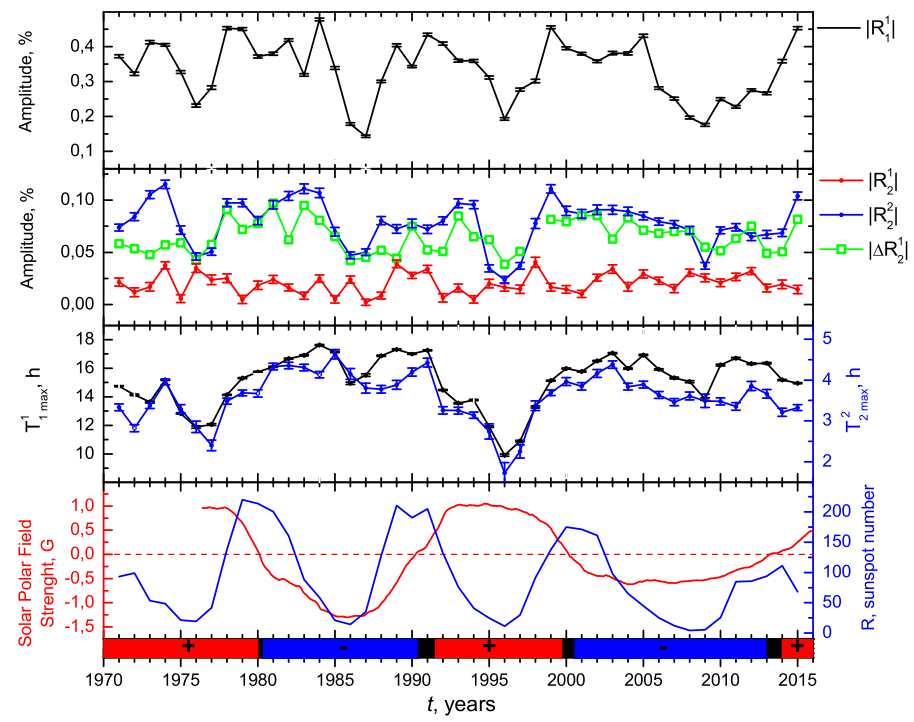

Figure 2: The observed mean annual values of amplitudes of symmetric diurnal $\left|\vec{R}_{1}^{1}\right|$, antisymmetric diurnal $\left|\vec{R}_{2}^{1}\right|$, semidiurnal $\left|\vec{R}_{2}^{2}\right|$ and yearly swing of antisymmetric diurnal $\left|\triangle \vec{R}_{2}^{1}\right|$ variations of CR. Also the maximum times of symmetric diurnal $T_{1, \max }^{1}$ and semidiurnal $T_{2, \max }^{2}$ variations. The solar sunspot number and the average solar field of the Northern and Southern Hemispheres are shown

months the vector is oriented to $21 \mathrm{~h}$ and during the summer months - $9 \mathrm{~h}$. And the vector of semidiurnal variations $\vec{R}_{2}^{2}$ oscillates in more complicated way, rotating counter clockwise during a year and, as a rule, during the autumn months it has lesser amplitude. The value of yearly spread of the vector $\vec{R}_{2}^{1}$ experiences insignificant dependence with solar activity (see figure 2 ). In order to describe the obtained annual variations of $\vec{R}_{2}^{1}$ and $\vec{R}_{2}^{2}$ we have used the mechanisms of tensor anisotropy formation: the screening of CR by interplanetary magnetic field [9] and the shear flow of solar wind [10]. Let us represent the tensor anisotropy of CR as a summ of both mentioned

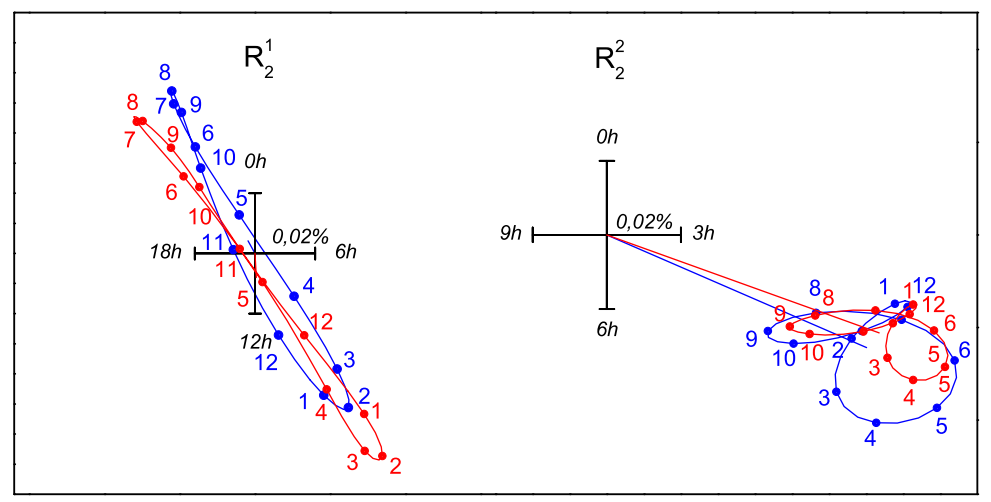

Figure 3: The vector diagrams of the observed (blue curve) and expected by the model (red curve) annual changes of the components of tensor anisotropy: antisymmetric diurnal $\vec{R}_{2}^{1}$ and semidiurnal $\vec{R}_{2}^{2}$ variations of CR. On hour dials the ends of the vectors are connected by solid curves, the numbers near the points indicates months 
mechanisms. In the heliographic coordinate system, which polar axis is directed along north pole of the Sun and zero longitude corresponds to the position of the Sun, the screening mechanism will have the components

$$
\vec{A}_{1}=(1 / 2,0,0,(\sqrt{3} / 2) \cos 2 \phi,(\sqrt{3} / 2) \sin 2 \phi),
$$

where $\phi$ is an angle of deflection between the archimendian spiral and radial direction.

In the same coordinate system the shear flow mechanism will give:

$$
\vec{A}_{2}=(0, \cos \psi, \sin \psi, 0,0),
$$

where $\psi$ defines the direction of increment of current speed.

In order to accurately balance our model and observations it is required to suggest that the value of screening mechanism $\vec{A}_{1}$ depends on the heliolatitude of the Earth and therefore on the time of year. The next factor have to be added:

$$
h(t)=1-k_{1} \sin t-k_{2}\left(\sin ^{2} t-1 / 2\right),
$$

where $t$ is heliolatitude of the Earth that counts from the 7th of July, when the Earth is in the solar equator, $k_{1}$ and $k_{2}$ are free parameters.

Now the final vector of anisotropy

$$
\vec{A}=h(t) \vec{A}_{1}+k_{3} \vec{A}_{2}
$$

can be converted to the terrestrial coordinate system, which polar axis is directed to the South pole of the Earth and zero longitude corresponds to the position of the Sun. Transition matrices are depends on heliolatitude angle $t$.

Balancing the model and the observed data is achieved for the values of angles $\phi=50^{\circ}$, $\psi=-40^{\circ}$ and coefficients $k_{1}=0.2, k_{2}=0.12$ and $k_{3}=0.05$ (figure 3 ). Low value of $k_{3}$ shows that the screening mechanism plays the major role. Presence of positive value $k_{1}$ indicates that the interplanetary magnetic field has a shift to the south heliolatitudes and the ratio between $k_{1}$ and $k_{2}$ allows to estimate this shift as $\approx 6.3^{\circ}$. The north-south asymmetry of the interplanetary magnetic field was earlier found and measured in works [11, 12], which systematically was directed to the south and during the periods of solar activity minima has the values $7-8^{\circ}$.

\section{Conclusions}

1. By the data of multidirectional muon telescope st. Nagoya for the period 1971-2015 using the method of receiving vectors the decomposition of the observed diurnal variations into the vector and tensor anisotropies of CR is made.

2. It is shown that the vector anisotropy of CR experiences changes with the solar magnetic cycle and solar activity. The main mechanism of the generation of this anisotropy is convectivediffusive and drift motion of CR. During the periods of solar activity minima in negative polarity of GMFS the influence of electrostatic potential of the heliosphere is found. 
3. It is shown that the components of tensor anisotropy $\left(a_{2}^{1}, b_{2}^{1}, a_{2}^{2}, b_{2}^{2}\right)$ experience stable annual and semiannual oscillations during the whole investigated time period, which are generated mainly by the CR screening mechanism. The mechanism of CR shear flow has a small contribution to this variations. Comparison of the model and experiment indicates that there is a shift of interplanetary magnetic field to the south of the solar equator on $\approx 6.3^{\circ}$.

\section{Acknowledgments}

The work was supported by the grants RFBR Nos. 15-42-05085-r_vostok_a, 15-42-05083-r_ vostok_a and the program of Presidium of SB RAS No. 23. We acknowledge Cosmic Ray Section, Solar-Terrestrial Environment Laboratory, Nagoya University and Wilcox Solar Observatory for providing the data.

\section{References}

[1] G.F. Krymsky, Diffusion mechanism of diurnal cosmic ray variations, Geomag. Aeron 4 (1964) 763

[2] E.N. Parker, The passage of energetic charged particles through interplanetary space, Planet. Space Sci 13 (1965) 9

[3] E.H. Levy, Theory of the Solar-Magnetic-Cycle Wave in the Diurnal Variation of Energetic Cosmic Rays: Physical Basis of the Anisotropy, JGR 81 (1976) 2082

[4] G.F. Krymsky, P.A. Krivoshapkin, S.K. Gerasimova and P.Yu. Gololobov, Tensor anisotropy of cosmic rays, Astron. Lett. 40 (2014) 230

[5] P.Yu. Gololobov, G.F. Krymsky, P.A. Krivoshapkin, S.K. Gerasimova, V.G. Grigoryev, Tensor anisotropy of cosmic rays by data of neutron monitors and muon telescopes, J. Phys.: Conf. Ser. 632 (2015) 012056

[6] K. Fujimoto, A. Inoue, K. Murakami et al., Coupling coefficients of cosmic ray daily variations for meson telescopes, Report of cosmic ray research laboratory 9 (1984)

[7] K. Munakata, M. Kozai, C. Kato, J. Kota, Long-term variation of the solar diurnal anisotropy of galactic cosmic rays observed with the Nagoya multidirectional muon detector, The Astrophysical Journal 791 (2014) 16

[8] S.K. Gerasimova, P.Yu. Gololobov, P.A. Krivoshapkin, G.F. Krymsky, Studying semidiurnal variations of cosmic rays, Bull. of the RAS: Physics 77 (2013) 526

[9] P.A. Krivoshapkin, G.F. Krymsky, A.I. Kuzmin, G.V. Skripin, E.A. Metlyaeva, The second spherical harmonics in the distribution of cosmic rays, Acta Physics Academiae Scientiarum Hungaricae 29 (1970) 147

[10] E.G. Berezhko, Acceleration of charged particles in a cosmic-phase shear flow, JETP Letters 33 (1981) 399

[11] G.F. Krymsky, P.A. Krivoshapkin, V.P. Mamrukova and S.K. Gerasimova, Heliospheric modulation of high-energy cosmic rays: I. Deformation of the neutral surface, JETP 104 (2007) 196

[12] G.F. Krymsky, P.A. Krivoshapkin, V.P. Mamrukova and S.K. Gerasimova, Anisotropy of high-energy cosmic rays, Astron. Lett. 36 (2010) 596 